\title{
A Unified Generalization of the Catalan, Fuss, and Fuss-Catalan Numbers
}

\author{
Feng Qi ${ }^{1,2,3, * \mathbb{D}}$, Xiao-Ting Shi ${ }^{2,4} \mathbb{D}$ and Pietro Cerone ${ }^{5} \mathbb{D}$ \\ 1 College of Mathematics, Inner Mongolia University for Nationalities, Tongliao 028043, China \\ 2 School of Mathematical Sciences, Tianjin Polytechnic University, Tianjin 300387, China \\ 3 Institute of Mathematics, Henan Polytechnic University, Jiaozuo 454010, China \\ 4 Secondary School, Yingxiong Street, Fenyang, Lüliang 032200, China; xiao-ting.shi@hotmail.com \\ 5 Department of Mathematics and Statistics, La Trobe University, Melbourne, VIC 3086, Australia; \\ p.cerone@latrobe.edu.au or pcerone53@gmail.com \\ * Correspondence: qifeng618@gmail.com or qifeng618@hotmail.com
}

Received: 1 April 2019; Accepted: 5 May 2019; Published: 8 May 2019

\begin{abstract}
In the paper, the authors introduce a unified generalization of the Catalan numbers, the Fuss numbers, the Fuss-Catalan numbers, and the Catalan-Qi function, and discover some properties of the unified generalization, including a product-ratio expression of the unified generalization in terms of the Catalan-Qi functions, three integral representations of the unified generalization, and the logarithmically complete monotonicity of the second order for a special case of the unified generalization.
\end{abstract}

Keywords: unified generalization; Catalan number; Fuss number; Fuss-Catalan number; Catalan-Qi function; product-ratio expression; logarithmically complete monotonicity of the second order; integral representation

MSC: Primary 11B83; Secondary 11B75, 11R33, 11S23, 11Y35, 11Y55, 11Y60, 26A48, 26A51, 30E20, 33B15

\section{Introduction}

As well known from [1,2], Catalan numbers $C_{n}$ are used in the study of set partitions in different areas of mathematics. In particular, in combinatorial mathematics, the Catalan numbers $C_{n}$ form a sequence of natural numbers that occur in various counting problems, often involving recursively-defined objects. There are many counting problems in combinatorics whose solution is given by the Catalan numbers $C_{n}$. The book [3] contains a set of exercises which describe 66 different interpretations of the Catalan numbers.

The Catalan numbers $C_{n}$ can be generated by

$$
\frac{2}{1+\sqrt{1-4 x}}=\frac{1-\sqrt{1-4 x}}{2 x}=\sum_{n=0}^{\infty} C_{n} x^{n}=1+x+2 x^{2}+5 x^{3}+14 x^{4}+42 x^{5}+132 x^{6}+\cdots .
$$

One of the explicit formulas of $C_{n}$ for $n \geq 0$ reads that

$$
C_{n}=\frac{1}{n+1}\left(\begin{array}{c}
2 n \\
n
\end{array}\right)=\frac{4^{n} \Gamma(n+1 / 2)}{\sqrt{\pi} \Gamma(n+2)}
$$

where

$$
\Gamma(z)=\int_{0}^{\infty} t^{z-1} e^{-t} \mathrm{~d} t, \quad \Re(z)>0
$$


is the classical Euler gamma function. In [2,4,5] and ([1] pp. 110-111), it was mentioned that there exists an asymptotic expansion

$$
C_{x} \triangleq \frac{4^{x} \Gamma\left(x+\frac{1}{2}\right)}{\sqrt{\pi} \Gamma(x+2)} \sim \frac{4^{x}}{\sqrt{\pi}}\left(\frac{1}{x^{3 / 2}}-\frac{9}{8} \frac{1}{x^{5 / 2}}+\frac{145}{128} \frac{1}{x^{7 / 2}}+\cdots\right)
$$

for the Catalan function $C_{x}$. For new developments on (1), see [6] and the review paper in [7], in which there are plenty of closely related references.

A generalization of the Catalan numbers $C_{n}$ was defined in [8,9] by

$$
{ }_{p} d_{n}=\frac{1}{n}\left(\begin{array}{c}
p n \\
n-1
\end{array}\right)=\frac{1}{(p-1) n+1}\left(\begin{array}{c}
p n \\
n
\end{array}\right)
$$

for $n, p \geq 1$. It is obvious that $C_{n}={ }_{2} d_{n}$. In ([1] pp. 375-376), the generalization ${ }_{p+1} d_{n}$ of the Catalan numbers $C_{n}$ is denoted by $C(n, p)$ for $p \geq 0$ and is called as the generalized Catalan numbers. In ([1] pp. 377-378), the Fuss numbers

$$
F(m, n)=\frac{1}{m n+1}\left(\begin{array}{c}
m n+1 \\
n
\end{array}\right)
$$

were given and discussed. It is apparent that $F(2, n)=C_{n}$.

In combinatorial mathematics and statistics, the Fuss-Catalan numbers $A_{n}(p, r)$ are defined [10] as numbers of the form

$$
A_{n}(p, r)=\frac{r}{n p+r}\left(\begin{array}{c}
n p+r \\
n
\end{array}\right)=\frac{r \Gamma(n p+r)}{\Gamma(n+1) \Gamma(n(p-1)+r+1)} .
$$

It is easy to see that

$$
A_{n}(p, 1)=F(p, n), \quad A_{n}(2,1)=C_{n}, \quad n \geq 0
$$

and

$$
A_{n-1}(p, p)={ }_{p} d_{n}=C(n, p-1), \quad n, p \geq 1 .
$$

There have existed some literature, such as $[1,2,11-21]$, on the investigation of the Fuss-Catalan numbers $A_{n}(p, r)$.

In [22], an alternative and analytical generalization of the Catalan numbers $C_{n}$ and the Catalan function $C_{x}$ was introduced by

$$
C(a, b ; z)=\frac{\Gamma(b)}{\Gamma(a)}\left(\frac{b}{a}\right)^{z} \frac{\Gamma(z+a)}{\Gamma(z+b)}, \quad \Re(a), \Re(b)>0, \quad \Re(z) \geq 0 .
$$

For uniqueness and convenience of referring to the quantity $C(a, b ; z)$, we call the quantity $C(a, b ; z)$ the Catalan-Qi function and, when taking $z=n \geq 0$, call $C(a, b ; n)$ the Catalan-Qi numbers. It is not difficult to verify that $C\left(\frac{1}{2}, 2 ; n\right)=C_{n}$ and

$$
C(n+1,2 ;(m-1) n)=\left(\frac{2}{n+1}\right)_{m}^{(m-1) n} d_{n}=\left(\frac{2}{n+1}\right)^{(m-1) n} C(n, m-1)
$$

for $m, n \geq 1$. In [22], it was obtained that

$$
C(a, b ; z)=\frac{\Gamma(b)}{\Gamma(a)}\left(\frac{b}{a}\right)^{z} \frac{(z+a)^{z}}{(z+b)^{z+b-a}} \exp \left[b-a+\int_{0}^{\infty} \frac{1}{t}\left(\frac{1}{1-e^{-t}}-\frac{1}{t}-a\right)\left(e^{-a t}-e^{-b t}\right) e^{-z t} \mathrm{~d} t\right]
$$


for $\Re(a), \Re(b)>0$ and $\Re(z) \geq 0$. Recently, we discovered in ([23] Theorem 1.1) relations between the Fuss-Catalan numbers $A_{n}(p, r)$ and the Catalan-Qi numbers $C(a, b ; n)$, one of which reads that

$$
A_{n}(p, r)=r^{n} \frac{\prod_{k=0}^{p-1} C\left(\frac{k+r}{p}, 1 ; n\right)}{\prod_{k=0}^{p-2} C\left(\frac{k+r+1}{p-1}, 1 ; n\right)}
$$

for integers $n \geq 0, p>1$, and $r>0$. In recent papers [6,22-32], among other things, some properties, including the general expression and a generalization of the asymptotic expansion (1), the monotonicity, logarithmic convexity, (logarithmically) complete monotonicity, minimality, Schur-convexity, product and determinantal inequalities, exponential representations, integral representations, a generating function, connections with the Bessel polynomials and the Bell polynomials of the second kind, and identities, of the Catalan numbers $C_{n}$, the Catalan function $C_{x}$, the Catalan-Qi numbers $C(a, b ; n)$, the Catalan-Qi function $C(a, b ; z)$, and the Fuss-Catalan numbers $A_{n}(p, r)$ were established.

In this paper, we will introduce a unified generalization of the Catalan numbers $C_{n}$, the generalized Catalan numbers $C(n, k)$, the Fuss numbers $F(k, n)$, the Fuss-Catalan numbers $A_{n}(p, r)$, and the Catalan-Qi function $C(a, b ; z)$. Hereafter, we will find a product-ratio expression, similar to the product-ratio expression (4), of the unified generalization in terms of the Catalan-Qi function $C(a, b ; z)$. Furthermore, based on the integral representation (3), on the Gauss multiplication formula for the gamma function, and on an integral representation for the logarithm of the gamma function $\Gamma(z)$, we will derive three integral representations of the unified generalization. Finally, we will establish the logarithmically complete monotonicity of the second order for the unified generalization.

\section{A Unified Generalization of the Catalan and Other Numbers}

Does there exist a unified and analytic generalization of the Catalan numbers $C_{n}$, the Fuss numbers $F(m, n)=C(n, m)$, the Fuss-Catalan numbers $A_{n}(p, r)$, and the Catalan-Qi function $C(a, b ; z)$ ? What is it concretely? In the early morning of September 15th 2015, a unified generalization was framed out eventually and successfully, and which can be described by a five-variable function

$$
Q(a, b ; p, q ; z)=\frac{\Gamma(b)}{\Gamma(a)}\left(\frac{b}{a}\right)^{(q-p+1) z}[\Gamma(z+1)]^{q-p} \frac{\Gamma(p z+a)}{\Gamma(q z+b)}
$$

where $\Re(a), \Re(b)>0, \Re(p), \Re(q)>0$, and $\Re(z) \geq 0$. For uniqueness and convenience of referring to the quantity $Q(a, b ; p, q ; z)$, we call $Q(a, b ; p, q ; z)$ the Fuss-Catalan-Qi function and, when taking $z=n \geq 0$, call $Q(a, b ; p, q ; n)$ the Fuss-Catalan-Qi numbers.

It is easy to see that

$$
\begin{aligned}
& Q\left(\frac{1}{2}, 2 ; 1,1 ; n\right)=Q(1,2 ; 2,1 ; n)=C_{n}, \quad Q(r, r+1 ; p, p-1 ; n)=A_{n}(p, r), \\
& Q(p, p+1 ; p, p-1 ; n-1)={ }_{p} d_{n}=C(n, p-1), \quad Q(a, b ; 1,1 ; z)=C(a, b ; z) .
\end{aligned}
$$

Accordingly, the Fuss-Catalan-Qi function $Q(a, b ; p, q ; z)$ is a unified generalization of the Catalan numbers $C_{n}$, the generalized Catalan numbers $C(n, m)$, the Fuss numbers $F(m, n)$, the Fuss-Catalan numbers $A_{n}(p, r)$, and the Catalan-Qi function $C(a, b ; z)$.

It is easy to see that the Fuss-Catalan-Qi function $Q(a, b ; p, q ; z)$ meets

$$
Q(b, a ; p, q ; z)=\left(\frac{a}{b}\right)^{2(q-p) z} \frac{1}{Q(a, b ; q, p ; z)}
$$

and, when $p=q$ or $a=b$,

$$
Q(a, b ; q, p ; z) Q(b, a ; p, q ; z)=1
$$


If only interchanging the role of $a$ and $b$, then

$$
Q(a, b ; p, q ; z) Q(b, a ; p, q ; z)=\left\{\begin{array}{l}
\frac{R(a, b ; 2 p ; z)}{R(b, a ; 2 q ; z)} \\
S(a ; p, q ; z) S(b ; p, q ; z)
\end{array}\right.
$$

where

$$
R(a, b ; r ; z)=\frac{\Gamma(r z+a) \Gamma(r z+b)}{[\Gamma(z+1)]^{r}}
$$

and

$$
S(c ; p, q ; z)=[\Gamma(z+1)]^{q-p} \frac{\Gamma(p z+c)}{\Gamma(q z+c)} .
$$

If only swapping $p$ and $q$, then

$$
Q(a, b ; p, q ; z) Q(a, b ; q, p ; z)=\left\{\begin{array}{l}
\frac{F(b ; p, q ; z)}{F(a ; p, q ; z)} \\
G(a, b ; p ; z) G(a, b ; q ; z),
\end{array}\right.
$$

where

$$
F(c ; p, q ; z)=\frac{\left[c^{z} \Gamma(c)\right]^{2}}{\Gamma(p z+c) \Gamma(q z+c)}
$$

and

$$
G(a, b ; r ; z)=\frac{\Gamma(b)}{\Gamma(a)}\left(\frac{b}{a}\right)^{z} \frac{\Gamma(r z+a)}{\Gamma(r z+b)} .
$$

\section{A Product-Ratio Expression of the Fuss-Catalan-Qi Function}

Motivated by the product-ratio expression (4), we now find out a product-ratio expression of the Fuss-Catalan-Qi function $Q(a, b ; p, q ; z)$.

Theorem 1. For $\Re(a), \Re(b)>0$ and $\Re(z) \geq 0$, when $p, q \in \mathbb{N}$, we have

$$
Q(a, b ; p, q ; z)=\left[\left(\frac{b}{a}\right)^{q-p+1} \frac{\Gamma(b) \Gamma(p+a)}{\Gamma(a) \Gamma(q+b)}\right]^{z} \frac{\prod_{k=0}^{p-1} C\left(\frac{k+a}{p}, 1 ; z\right)}{\prod_{k=0}^{q-1} C\left(\frac{k+b}{q}, 1 ; z\right)} .
$$

Proof. By the Gauss multiplication formula

$$
\Gamma(n z)=\frac{n^{n z-1 / 2}}{(2 \pi)^{(n-1) / 2}} \prod_{k=0}^{n-1} \Gamma\left(z+\frac{k}{n}\right), \quad n \in \mathbb{N}
$$

in ([33] p. 256, 6.1.20), the Fuss-Catalan-Qi function $Q(a, b ; p, q ; z)$ can be written as

$$
\begin{aligned}
& Q(a, b ; p, q ; z)=\frac{\Gamma(b)}{\Gamma(a)}\left(\frac{b}{a}\right)^{(q-p+1) z}[\Gamma(z+1)]^{q-p} \frac{\Gamma\left(p\left(z+\frac{a}{p}\right)\right)}{\Gamma\left(q\left(z+\frac{b}{q}\right)\right)} \\
& =\frac{\Gamma(b)}{\Gamma(a)}\left(\frac{b}{a}\right)^{(q-p+1) z}[\Gamma(z+1)]^{q-p} \frac{\frac{p^{p z+a-1 / 2}}{(2 \pi)^{(p-1) / 2}} \prod_{k=0}^{p-1} \Gamma\left(z+\frac{k+a}{p}\right)}{\frac{q^{q z+b-1 / 2}}{(2 \pi)^{(q-1) / 2}} \prod_{k=0}^{q-1} \Gamma\left(z+\frac{k+b}{q}\right)}
\end{aligned}
$$




$$
\begin{aligned}
& =\frac{\Gamma(b)}{\Gamma(a)}\left(\frac{b}{a}\right)^{(q-p+1) z} \frac{\frac{p^{p z+a-1 / 2}}{(2 \pi)^{(p-1) / 2}} \prod_{k=0}^{p-1} \frac{\Gamma\left(z+\frac{k+a}{p}\right)}{\Gamma(z+1)}}{\frac{q^{q z+b-1 / 2}}{(2 \pi)^{(q-1) / 2}} \prod_{k=0}^{q-1} \frac{\Gamma\left(z+\frac{k+b}{q}\right)}{\Gamma(z+1)}} \\
& =\frac{\Gamma(b)}{\Gamma(a)}\left(\frac{b}{a}\right)^{(q-p+1) z} \frac{\prod_{k=0}^{q-1} \frac{\Gamma(1)}{\Gamma\left(\frac{k+b}{q}\right)}\left(\frac{q}{k+b}\right)^{z} \frac{p^{p z+a-1 / 2}}{(2 \pi)^{(p-1) / 2}} \prod_{k=0}^{p-1} \frac{\Gamma(1)}{\Gamma\left(\frac{k+a}{p}\right)}\left(\frac{p}{k+a}\right)^{z} \frac{\Gamma\left(z+\frac{k+a}{p}\right)}{\Gamma(z+1)}}{\prod_{k=0}^{p-1} \frac{\Gamma(1)}{\Gamma\left(\frac{k+a}{p}\right)}\left(\frac{p}{k+a}\right)^{z} \frac{q^{q z+b-1 / 2}}{(2 \pi)^{(q-1) / 2}} \prod_{k=0}^{q-1} \frac{\Gamma(1)}{\Gamma\left(\frac{k+b}{q}\right)}\left(\frac{q}{k+b}\right)^{z} \frac{\Gamma\left(z+\frac{k+b}{q}\right)}{\Gamma(z+1)}} \\
& =\frac{\Gamma(b)}{\Gamma(a)}\left(\frac{b}{a}\right)^{(q-p+1) z} \frac{\prod_{k=0}^{p-1} \Gamma\left(\frac{a}{p}+\frac{k}{p}\right)}{\prod_{k=0}^{q-1} \Gamma\left(\frac{b}{q}+\frac{k}{q}\right)} \frac{\prod_{k=0}^{p-1}(k+a)^{z}}{\prod_{k=0}^{q-1}(k+b)^{z}} \frac{q^{z q}}{p^{z p}} \frac{\frac{p^{p z+a-1 / 2}}{(2 \pi)^{(p-1) / 2}} \prod_{k=0}^{p-1} C\left(\frac{k+a}{p}, 1 ; z\right)}{\frac{q^{q z+b-1 / 2}}{(2 \pi)^{(q-1) / 2}} \prod_{k=0}^{q-1} C\left(\frac{k+b}{q}, 1 ; z\right)} \\
& =\frac{\Gamma(b)}{\Gamma(a)}\left(\frac{b}{a}\right)^{(q-p+1) z} \frac{\Gamma(a) \frac{(2 \pi)^{(p-1) / 2}}{p^{a-1 / 2}}}{\Gamma(b) \frac{(2 \pi)^{(q-1) / 2}}{q^{b-1 / 2}}}\left[\frac{\prod_{k=0}^{p-1}(k+a)}{\prod_{k=0}^{q-1}(k+b)}\right]^{z} \frac{p^{a-1 / 2}}{(2 \pi)^{(p-1) / 2}} \frac{(2 \pi)^{(q-1) / 2}}{q^{b-1 / 2}} \frac{\prod_{k=0}^{p-1} C\left(\frac{k+a}{p}, 1 ; z\right)}{\prod_{k=0}^{q-1} C\left(\frac{k+b}{q}, 1 ; z\right)} \\
& =\left(\frac{b}{a}\right)^{(q-p+1) z}\left[\frac{\prod_{k=0}^{p-1}(k+a)}{\prod_{k=0}^{q-1}(k+b)}\right]^{z} \frac{\prod_{k=0}^{p-1} C\left(\frac{k+a}{p}, 1 ; z\right)}{\prod_{k=0}^{q-1} C\left(\frac{k+b}{q}, 1 ; z\right)} \\
& =\left(\frac{b}{a}\right)^{(q-p+1) z}\left[\frac{\Gamma(b) \Gamma(p+a)}{\Gamma(a) \Gamma(q+b)}\right]^{z} \frac{\prod_{k=0}^{p-1} C\left(\frac{k+a}{p}, 1 ; z\right)}{\prod_{k=0}^{q-1} C\left(\frac{k+b}{q}, 1 ; z\right)} .
\end{aligned}
$$

The identity (7) is thus proved. The proof of Theorem 1 is complete.

Remark 1. Before getting (7), we did not appreciate the analytic meanings of the form of the product-ratio expression (4) because before catching sight of the unified generalization (5), we did not appreciate the analytic meanings of the form of the Fuss-Catalan numbers $A_{n}(p, r)$ in (2).

Remark 2. From (6) and (7), we derive the identity (4) and

$$
p d_{n}=C(n, p-1)=p^{n-1} \frac{\prod_{k=0}^{p-1} C\left(1+\frac{k}{p}, 1 ; n-1\right)}{\prod_{k=0}^{p-2} C\left(1+\frac{k+2}{p-1}, 1 ; n-1\right)} .
$$

Remark 3. When $p=q$, the product-ratio expression (7) can be reformulated as

$$
Q(a, b ; q, q ; z)=\frac{K(a, q, z)}{K(b, q, z)},
$$

where

$$
K(c, q, z)=\left[\frac{\Gamma(q+c)}{\Gamma(1+c)}\right] \prod_{k=0}^{z q-1} C\left(\frac{k+c}{q}, 1 ; z\right)
$$

If taking $a=b$, then

$$
Q(a, a ; p, q ; z)=\frac{\frac{\Gamma(p z+a)}{[\Gamma(z+1)]^{p}}}{\frac{\Gamma(q z+a)}{[\Gamma(z+1)]^{q}}}=\frac{[\Gamma(p+a)]^{z} \prod_{k=0}^{p-1} C\left(\frac{k+a}{p}, 1 ; z\right)}{[\Gamma(q+a)]^{z} \prod_{k=0}^{q-1} C\left(\frac{k+a}{q}, 1 ; z\right)} .
$$




\section{Integral Representations of the Fuss-Catalan-Qi Function}

Making use of the integral representation (3) and the product-ratio expression (7), we now derive the first integral representation of the Fuss-Catalan-Qi function $Q(a, b ; p, q ; z)$.

Theorem 2. For $\Re(a), \Re(b)>0$, and $\Re(z) \geq 0$, when $p, q \in \mathbb{N}$, we have

$$
\begin{aligned}
Q(a, b ; p, q ; z)= & (2 \pi)^{(q-p) / 2} \frac{\Gamma(b)}{\Gamma(a)} \frac{p^{a-1 / 2}}{q^{b-1 / 2}}(z+1)^{(q-p)(z+1 / 2)+(a-b)} \\
& \times\left[\left(\frac{b}{a}\right)^{q-p+1} \frac{\prod_{k=0}^{p-1}(a+p z+k)}{\prod_{k=0}^{q-1}(b+q z+k)}\right]^{z} \exp \left\{\frac{p-q}{2}+(b-a)\right. \\
& +\int_{0}^{\infty} \frac{e^{-z t}}{t}\left[\left(\frac{1}{1-e^{-t}}-\frac{1}{t}-\frac{a}{p}\right)\left(\frac{1-e^{-t}}{1-e^{-t / p}} e^{-a t / p}-p e^{-t}\right)\right. \\
& -\left(\frac{1}{1-e^{-t}}-\frac{1}{t}-\frac{b}{q}\right)\left(\frac{1-e^{-t}}{1-e^{-t / q}} e^{-b t / q}-q e^{-t}\right) \\
& -\left(\frac{e^{-t / p}}{p\left(1-e^{-t / p}\right)}-\frac{e^{-t}}{1-e^{-t}}\right) \frac{1-e^{-t}}{1-e^{-t / p}} e^{-a t / p} \\
& \left.\left.+\left(\frac{e^{-t / q}}{q\left(1-e^{-t / q}\right)}-\frac{e^{-t}}{1-e^{-t}}\right) \frac{1-e^{-t}}{1-e^{-t / q}} e^{-b t / q}+\frac{p-q}{2} e^{-t}\right] \mathrm{~d} t\right\} .
\end{aligned}
$$

Proof. Making use of the integral representation (3) leads to

$$
\begin{aligned}
& \prod_{k=0}^{p-1} C\left(\frac{k+a}{p}, 1 ; z\right)=\prod_{k=0}^{p-1}\left[\frac{1}{\Gamma\left(\frac{k+a}{p}\right)}\left(\frac{p}{k+a}\right)^{z} \frac{\left(z+\frac{k+a}{p}\right)^{z}}{(z+1)^{z+1-\frac{k+a}{p}}}\right] \\
& \times \exp \left[\sum_{k=0}^{p-1}\left(1-\frac{k+a}{p}\right)+\sum_{k=0}^{p-1} \int_{0}^{\infty} \frac{1}{t}\left(\frac{1}{1-e^{-t}}-\frac{1}{t}-\frac{k+a}{p}\right)\left(e^{-\frac{k+a}{p} t}-e^{-t}\right) e^{-z t} \mathrm{~d} t\right] \\
& =\frac{1}{\prod_{k=0}^{p-1} \Gamma\left(\frac{k+a}{p}\right)} \frac{1}{\left[\prod_{k=0}^{p-1}(k+a)\right]^{z}} \frac{\prod_{k=0}^{p-1}(p z+k+a)^{z}}{(z+1)^{p(z+1)}}(z+1)^{a+\frac{\sum_{k=0}^{p-1} k}{p}} \\
& \times \exp \left[p-a-\frac{\sum_{k=0}^{p-1} k}{p}+\int_{0}^{\infty} \frac{e^{-z t}}{t} \sum_{k=0}^{p-1}\left(\frac{1}{1-e^{-t}}-\frac{1}{t}-\frac{k+a}{p}\right)\left(e^{-\frac{k+a}{p} t}-e^{-t}\right) \mathrm{d} t\right] \\
& =\frac{1}{\prod_{k=0}^{p-1} \Gamma\left(\frac{a}{p}+\frac{k}{p}\right)}\left[\frac{\Gamma(a)}{\Gamma(p+a)}\right]^{z}\left[\frac{\Gamma(p(z+1)+a)}{\Gamma(p z+a)}\right]^{z} \frac{(z+1)^{a+(p-1) / 2}}{(z+1)^{p(z+1)}} \\
& \times \exp \left[p-a-\frac{p-1}{2}+\int_{0}^{\infty} \frac{e^{-z t}}{t} \sum_{k=0}^{p-1}\left(\frac{1}{1-e^{-t}}-\frac{1}{t}-\frac{k+a}{p}\right)\left(e^{-\frac{k+a}{p} t}-e^{-t}\right) \mathrm{d} t\right] \\
& =\frac{p^{a-1 / 2}}{(2 \pi)^{(p-1) / 2} \Gamma(a)}\left[\frac{\Gamma(a) \Gamma(p)}{\Gamma(p+a)}\right]^{z}\left[\frac{\Gamma(p(z+1)+a)}{\Gamma(p z+a) \Gamma(p)}\right]^{z} \frac{1}{(z+1)^{p(z+1 / 2)-a+1 / 2}} \\
& \times \exp \left\{\frac{p}{2}-a+\frac{1}{2}+\int_{0}^{\infty} \frac{e^{-z t}}{t}\left[\left(\frac{1}{1-e^{-t}}-\frac{1}{t}-\frac{a}{p}\right)\left(\sum_{k=0}^{p-1} e^{-\frac{k+a}{p} t}-p e^{-t}\right)\right.\right. \\
& \left.\left.-\sum_{k=0}^{p-1} \frac{k}{p} e^{-\frac{k+a}{p} t}+\frac{p-1}{2} e^{-t}\right] \mathrm{~d} t\right\} \\
& =\frac{p^{a-1 / 2}}{(2 \pi)^{(p-1) / 2} \Gamma(a)}\left[\frac{B(a, p)}{B(a+p z, p)}\right]^{z} \frac{1}{(z+1)^{p(z+1 / 2)-a+1 / 2}} \\
& \times \exp \left\{\frac{p}{2}-a+\frac{1}{2}+\int_{0}^{\infty} \frac{e^{-z t}}{t}\left[\left(\frac{1}{1-e^{-t}}-\frac{1}{t}-\frac{a}{p}\right)\left(\sum_{k=0}^{p-1} e^{-\frac{k+a}{p} t}-p e^{-t}\right)\right.\right.
\end{aligned}
$$




$$
\begin{gathered}
\left.\left.-\sum_{k=0}^{p-1} \frac{k}{p} e^{-\frac{k+a}{p} t}+\frac{p-1}{2} e^{-t}\right] \mathrm{~d} t\right\} \\
=\frac{p^{a-1 / 2}}{(2 \pi)^{(p-1) / 2} \Gamma(a)}\left[\frac{B(a, p)}{B(a+p z, p)}\right]^{z} \frac{1}{(z+1)^{p(z+1 / 2)-a+1 / 2}} \\
\times \exp \left\{\frac{p}{2}-a+\frac{1}{2}+\int_{0}^{\infty} \frac{e^{-z t}}{t}\left[\left(\frac{1}{1-e^{-t}}-\frac{1}{t}-\frac{a}{p}\right)\left(e^{-a t / p} \frac{1-e^{-t}}{1-e^{-t / p}}-p e^{-t}\right)\right.\right. \\
\left.\left.-\frac{p\left(e^{-t / p}-1\right) e^{-t}+\left(1-e^{-t}\right) e^{-t / p}}{p\left(1-e^{-t / p}\right)^{2}} e^{-a t / p}+\frac{p-1}{2} e^{-t}\right] \mathrm{~d} t\right\},
\end{gathered}
$$

where $B(p, q)=\frac{\Gamma(p) \Gamma(q)}{\Gamma(p+q)}$ is the classical beta function. Similarly, we also have

$$
\begin{gathered}
\prod_{k=0}^{q-1} C\left(\frac{k+b}{q}, 1 ; z\right)=\frac{q^{b-1 / 2}}{(2 \pi)^{(q-1) / 2} \Gamma(b)}\left[\frac{B(b, q)}{B(b+q z, q)}\right]^{z} \frac{1}{(z+1)^{q(z+1 / 2)-b+1 / 2}} \\
\times \exp \left\{\frac{q}{2}-b+\frac{1}{2}+\int_{0}^{\infty} \frac{e^{-z t}}{t}\left[\left(\frac{1}{1-e^{-t}}-\frac{1}{t}-\frac{b}{q}\right)\left(e^{-b t / q} \frac{1-e^{-t}}{1-e^{-t / q}}-q e^{-t}\right)\right.\right. \\
\left.\left.-\frac{q\left(e^{-t / q}-1\right) e^{-t}+\left(1-e^{-t}\right) e^{-t / q}}{q\left(1-e^{-t / q}\right)^{2}} e^{-b t / q}+\frac{q-1}{2} e^{-t}\right] \mathrm{~d} t\right\} .
\end{gathered}
$$

Consequently, we obtain

$$
\begin{aligned}
\frac{\prod_{k=0}^{p-1} C\left(\frac{k+a}{p}, 1 ; z\right)}{\prod_{k=0}^{q-1} C\left(\frac{k+b}{q}, 1 ; z\right)}= & (2 \pi)^{(q-p) / 2}(z+1)^{(q-p)(z+1 / 2)+(a-b)} \frac{\Gamma(b)}{\Gamma(a)} \frac{p^{a-1 / 2}}{q^{b-1 / 2}} \\
& \times\left[\frac{B(a, p)}{B(a+p z, p)} \frac{B(b+q z, q)}{B(b, q)}\right]^{z} \exp \left\{\frac{p-q}{2}+(b-a)\right. \\
& +\int_{0}^{\infty} \frac{e^{-z t}}{t}\left[\left(\frac{1}{1-e^{-t}}-\frac{1}{t}-\frac{a}{p}\right)\left(e^{-a t / p} \frac{1-e^{-t}}{1-e^{-t / p}}-p e^{-t}\right)\right. \\
& -\left(\frac{1}{1-e^{-t}}-\frac{1}{t}-\frac{b}{q}\right)\left(e^{-b t / q} \frac{1-e^{-t}}{1-e^{-t / q}}-q e^{-t}\right) \\
& -\frac{p\left(e^{-t / p}-1\right) e^{-t}+\left(1-e^{-t}\right) e^{-t / p}}{p\left(1-e^{-t / p}\right)^{2}} e^{-a t / p} \\
& \left.\left.+\frac{q\left(e^{-t / q}-1\right) e^{-t}+\left(1-e^{-t}\right) e^{-t / q}}{q\left(1-e^{-t / q}\right)^{2}} e^{-b t / q}+\frac{p-q}{2} e^{-t}\right] \mathrm{~d} t\right\}
\end{aligned}
$$

Substituting this into (5) and simplifying yields the integral representation in Theorem 2. The proof of Theorem 2 is complete.

By the Gauss multiplication formula (8) and an integral representation for the logarithm of the gamma function $\Gamma(z)$, we can acquire the second integral representation of the Fuss-Catalan-Qi function $Q(a, b ; p, q ; z)$, which is seemingly simpler than the one in Theorem 2.

Theorem 3. For $a, b>0$ and $x \geq 0$, when $p, q \in \mathbb{N}$, we have

$$
\begin{aligned}
Q(a, b ; p, q ; x)= & (2 \pi)^{(q-p) / 2} e^{(p-q) / 2+b-a} \frac{\Gamma(b)}{\Gamma(a)}\left(\frac{b}{a}\right)^{(q-p+1) x} \\
& \times(x+1)^{(q-p)(x+1 / 2)} \frac{p^{p x+a-1 / 2}}{q^{q x+b-1 / 2}} \frac{\prod_{k=0}^{p-1}\left(x+\frac{a+k}{p}\right)^{x+(a+k) / p-1 / 2}}{\prod_{k=0}^{q-1}\left(x+\frac{b+k}{q}\right)^{x+(b+k) / q-1 / 2}}
\end{aligned}
$$




$$
\times \exp \left\{\int_{0}^{\infty} \beta(t)\left[(q-p) e^{-t}+\frac{1-e^{-t}}{1-e^{-t / p}} e^{-a t / p}-\frac{1-e^{-t}}{1-e^{-t / q}} e^{-b t / q}\right] e^{-x t} \mathrm{~d} t\right\}
$$

where

$$
\beta(t)=\frac{1}{t}\left(\frac{1}{e^{t}-1}-\frac{1}{t}+\frac{1}{2}\right)
$$

Proof. By Formula (8) and

$$
\ln \Gamma(z)=\ln \left(\sqrt{2 \pi} z^{z-1 / 2} e^{-z}\right)+\int_{0}^{\infty} \beta(t) e^{-z t} \mathrm{~d} t
$$

in ([34] (3.22)), we have

$$
\begin{aligned}
& \ln \left([\Gamma(x+1)]^{q-p} \frac{\Gamma(p x+a)}{\Gamma(q x+b)}\right)=(q-p) \ln \Gamma(x+1)+\ln \frac{\Gamma\left(p\left(x+\frac{a}{p}\right)\right)}{\Gamma\left(q\left(x+\frac{b}{q}\right)\right)} \\
& =(q-p) \ln \Gamma(x+1)+\ln \frac{\frac{p^{p x+a-1 / 2}}{(2 \pi)^{(p-1) / 2}} \prod_{k=0}^{p-1} \Gamma\left(x+\frac{a+k}{p}\right)}{\frac{q^{q x+b-1 / 2}}{(2 \pi)^{(q-1) / 2}} \prod_{k=0}^{q-1} \Gamma\left(x+\frac{b+k}{q}\right)} \\
& =(q-p) \ln \Gamma(x+1)+\ln \left[(2 \pi)^{(q-p) / 2} \frac{p^{p x+a-1 / 2}}{q^{q x+b-1 / 2}}\right] \\
& +\sum_{k=0}^{p-1} \ln \Gamma\left(x+\frac{a+k}{p}\right)-\sum_{k=0}^{q-1} \ln \Gamma\left(x+\frac{b+k}{q}\right) \\
& =(q-p) \ln \left[\sqrt{2 \pi}(x+1)^{x+1 / 2} e^{-(x+1)}\right]+(q-p) \int_{0}^{\infty} \beta(t) e^{-(x+1) t} \mathrm{~d} t \\
& +\ln \left[(2 \pi)^{(q-p) / 2} \frac{p^{p x+a-1 / 2}}{q^{q x+b-1 / 2}}\right]-\sum_{k=0}^{q-1} \int_{0}^{\infty} \beta(t) e^{-[x+(b+k) / q] t} \mathrm{~d} t \\
& +\sum_{k=0}^{p-1} \ln \left[\sqrt{2 \pi}\left(x+\frac{a+k}{p}\right)^{x+(a+k) / p-1 / 2} e^{-[x+(a+k) / p]}\right] \\
& -\sum_{k=0}^{q-1} \ln \left[\sqrt{2 \pi}\left(x+\frac{b+k}{q}\right)^{x+(b+k) / q-1 / 2} e^{-[x+(b+k) / q]}\right]+\sum_{k=0}^{p-1} \int_{0}^{\infty} \beta(t) e^{-[x+(a+k) / p] t} \mathrm{~d} t \\
& =\ln \left[(2 \pi)^{(q-p) / 2} \frac{p^{p x+a-1 / 2}}{q^{q x+b-1 / 2}}(x+1)^{(q-p)(x+1 / 2)} \frac{\prod_{k=0}^{p-1}\left(x+\frac{a+k}{p}\right)^{x+(a+k) / p-1 / 2}}{\prod_{k=0}^{q-1}\left(x+\frac{b+k}{q}\right)^{x+(b+k) / q-1 / 2}}\right] \\
& +(p-q)(x+1)-\sum_{k=0}^{p-1}\left(x+\frac{a+k}{p}\right)+\sum_{k=0}^{q-1}\left(x+\frac{b+k}{q}\right) \\
& +(q-p) \int_{0}^{\infty} \beta(t) e^{-(x+1) t} \mathrm{~d} t+\int_{0}^{\infty} \beta(t) e^{-(x+a / p) t} \sum_{k=0}^{p-1} e^{-k t / p} \mathrm{~d} t \\
& -\int_{0}^{\infty} \beta(t) e^{-(x+b / q) t} \sum_{k=0}^{q-1} e^{-k t / q} \mathrm{~d} t \\
& =\ln \left[(2 \pi)^{(q-p) / 2} \frac{p^{p x+a-1 / 2}}{q^{q x+b-1 / 2}}(x+1)^{(q-p)(x+1 / 2)} \frac{\prod_{k=0}^{p-1}\left(x+\frac{a+k}{p}\right)^{x+(a+k) / p-1 / 2}}{\prod_{k=0}^{q-1}\left(x+\frac{b+k}{q}\right)^{x+(b+k) / q-1 / 2}}\right] \\
& +\frac{p-q}{2}+b-a+(q-p) \int_{0}^{\infty} \beta(t) e^{-(x+1) t} \mathrm{~d} t \\
& +\int_{0}^{\infty} \beta(t) e^{-(x+a / p) t} \frac{1-e^{-t}}{1-e^{-t / p}} \mathrm{~d} t-\int_{0}^{\infty} \beta(t) e^{-(x+b / q) t} \frac{1-e^{-t}}{1-e^{-t / q}} \mathrm{~d} t
\end{aligned}
$$




$$
\begin{aligned}
= & \ln \left[(2 \pi)^{(q-p) / 2} \frac{p^{p x+a-1 / 2}}{q^{q x+b-1 / 2}}(x+1)^{(q-p)(x+1 / 2)} \frac{\prod_{k=0}^{p-1}\left(x+\frac{a+k}{p}\right)^{x+(a+k) / p-1 / 2}}{\prod_{k=0}^{q-1}\left(x+\frac{b+k}{q}\right)^{x+(b+k) / q-1 / 2}}\right] \\
& +\frac{p-q}{2}+b-a+\int_{0}^{\infty} \beta(t)\left[(q-p) e^{-t}+\frac{1-e^{-t}}{1-e^{-t / p}} e^{-a t / p}-\frac{1-e^{-t}}{1-e^{-t / q}} e^{-b t / q}\right] e^{-x t} \mathrm{~d} t .
\end{aligned}
$$

Substituting this into (5) leads to the integral representation (9).

Only by the integral representation (11), we can establish the third integral representation of the Fuss-Catalan-Qi function $Q(a, b ; p, q ; z)$, which is seemingly simpler than the previous ones.

Theorem 4. For $a, b, p, q>0$ and $x \geq 0$, we have

$$
\begin{array}{r}
Q(a, b ; p, q ; x)=\frac{\Gamma(b)}{\Gamma(a)}(2 \pi)^{(q-p) / 2} e^{p-q+b-a}\left(\frac{b}{a}\right)^{(q-p+1) x}(x+1)^{(q-p)(x+1 / 2)} \frac{(p x+a)^{p x+a-1 / 2}}{(q x+b)^{q x+b-1 / 2}} \\
\times \exp \left\{\int_{0}^{\infty} \beta(t)\left[(q-p) e^{-(x+1) t}-e^{-(q x+b) t}+e^{-(p x+a) t}\right] \mathrm{d} t\right\},
\end{array}
$$

where $\beta(t)$ is defined by (10).

Proof. By virtue of (11), we obtain

$$
\begin{gathered}
\ln \left([\Gamma(x+1)]^{q-p} \frac{\Gamma(p x+a)}{\Gamma(q x+b)}\right)=(q-p) \ln \Gamma(x+1)+\ln \Gamma(p x+a)-\ln \Gamma(q x+b) \\
=(q-p) \ln \left[\sqrt{2 \pi}(x+1)^{x+1 / 2} e^{-(x+1)}\right]+(q-p) \int_{0}^{\infty} \beta(t) e^{-(x+1) t} \mathrm{~d} t \\
+\ln \left[\sqrt{2 \pi}(p x+a)^{p x+a-1 / 2} e^{-(p x+a)}\right]+\int_{0}^{\infty} \beta(t) e^{-(p x+a) t} \mathrm{~d} t \\
-\ln \left[\sqrt{2 \pi}(q x+b)^{q x+b-1 / 2} e^{-(q x+b)}\right]-\int_{0}^{\infty} \beta(t) e^{-(q x+b) t} \mathrm{~d} t \\
=p-q+b-a+\ln \left[(2 \pi)^{(q-p) / 2}(x+1)^{(q-p)(x+1 / 2)} \frac{(p x+a)^{p x+a-1 / 2}}{(q x+b)^{q x+b-1 / 2}}\right] \\
\quad+\int_{0}^{\infty} \beta(t)\left[(q-p) e^{-(x+1) t}+e^{-(p x+a) t}-e^{-(q x+b) t}\right] \mathrm{d} t .
\end{gathered}
$$

Substituting this into (5) leads to the integral representation (12). The proof of Theorem 4 is complete.

Remark 4. From (6) and the integral representation in Theorem 2, we obtain

$$
\begin{aligned}
A_{n} & (p, r)=\frac{1}{\sqrt{2 \pi}} r \frac{p^{r-1 / 2}}{(p-1)^{r+1 / 2}} \frac{1}{(n+1)^{n+3 / 2}}\left[\frac{\prod_{k=0}^{p-1}(k+r+p v)}{\prod_{k=0}^{p-2}(k+r+(p-1) v+1)}\right]^{n} \\
& \times \exp \left\{\frac{3}{2}+\int_{0}^{\infty} \frac{e^{-v t}}{t}\left[\left(\frac{1}{1-e^{-t}}-\frac{1}{t}-\frac{r}{p}\right)\left(e^{-\frac{r}{p} t} \frac{1-e^{-t}}{1-e^{-t / p}}-p e^{-t}\right)\right.\right. \\
& -\left(\frac{1}{1-e^{-t}}-\frac{1}{t}-\frac{r+1}{p-1}\right)\left(e^{-\frac{r+1}{p-1} t} \frac{1-e^{-t}}{1-e^{-t /(p-1)}}-(p-1) e^{-t}\right) \\
& -\left(\frac{e^{-t / p}}{p\left(1-e^{-t / p}\right)}-\frac{e^{-t}}{1-e^{-t}}\right) \frac{1-e^{-t}}{1-e^{-t / p}} e^{-\frac{r}{p} t} \\
& \left.\left.+\left(\frac{e^{-t /(p-1)}}{(p-1)\left(1-e^{-t /(p-1)}\right)}-\frac{e^{-t}}{1-e^{-t}}\right) \frac{1-e^{-t}}{1-e^{-t /(p-1)}} e^{-\frac{r+1}{p-1} t}+\frac{1}{2} e^{-t}\right] \mathrm{~d} t\right\}
\end{aligned}
$$


and

$$
\begin{aligned}
C & (n, p-1)=\frac{1}{\sqrt{2 \pi}}\left(\frac{p}{p-1}\right)^{p+1 / 2} \frac{1}{(n+1)^{n+1 / 2}}\left[\frac{\prod_{k=1}^{p-1}(k+p n)}{\prod_{k=0}^{p-2}(k+(p-1) n+2)}\right]^{n} \\
& \times \exp \left\{\frac{3}{2}+\int_{0}^{\infty} \frac{e^{-(n-1) t}}{t}\left[\left(\frac{1}{1-e^{-t}}-\frac{1}{t}-1\right)\left(\frac{1-e^{-t}}{1-e^{-t / p}}-p\right) e^{-t}\right.\right. \\
& -\left(\frac{1}{1-e^{-t}}-\frac{1}{t}-\frac{p+1}{p-1}\right)\left(e^{-\frac{p+1}{p-1} t} \frac{1-e^{-t}}{1-e^{-t /(p-1)}}-(p-1) e^{-t}\right) \\
& -\left(\frac{e^{-t / p}}{p\left(1-e^{-t / p)}\right.}-\frac{e^{-t}}{1-e^{-t}}\right) \frac{1-e^{-t}}{1-e^{-t / p}} e^{-t} \\
& \left.\left.+\left(\frac{e^{-t /(p-1)}}{(p-1)\left(1-e^{-t /(p-1)}\right)}-\frac{e^{-t}}{1-e^{-t}}\right) \frac{1-e^{-t}}{1-e^{-t /(p-1)}} e^{-\frac{p+1}{p-1} t}+\frac{1}{2} e^{-t}\right] \mathrm{~d} t\right\} .
\end{aligned}
$$

Remark 5. When $p=q$, the integral representation in Theorem 2 is reduced to

$$
\begin{aligned}
& Q(a, b ; q, q ; z)=\frac{\Gamma(b)}{\Gamma(a)} q^{a-b}(z+1)^{a-b}\left[\frac{b}{a} \prod_{k=0}^{q-1} \frac{a+q z+k}{b+q z+k}\right]^{z} \\
& \quad \times \exp \left\{b-a+\int_{0}^{\infty} \frac{e^{-z t}}{t}\left[(a-b) e^{-t}+\left(\frac{b}{q} e^{-b t / q}-\frac{a}{q} e^{-a t / q}\right) \frac{1-e^{-t}}{1-e^{-t / q}}\right.\right. \\
& \left.\left.\quad+\left(\frac{1+e^{-t}}{1-e^{-t}}-\frac{e^{-t / q}}{q\left(1-e^{-t / q}\right)}-\frac{1}{t}\right) \frac{1-e^{-t}}{1-e^{-t / q}}\left(e^{-a t / q}-e^{-b t / q}\right)\right] \mathrm{d} t\right\} .
\end{aligned}
$$

Remark 6. By the integral representation (12) and the second relation in (6), we find

$$
\begin{aligned}
A_{n}(p, r)=\frac{e^{2}}{\sqrt{2 \pi}} r(n+1)^{-(n+1 / 2)} \frac{(p n+r)^{p n+r-1 / 2}}{[(p-1) n+r+1]^{(p-1) n+r+1 / 2}} & \\
& \quad \times \exp \left\{\int_{0}^{\infty} \beta(t)\left[e^{-(p n+r) t}-e^{-((p-1) n+r+1) t}-e^{-(n+1) t}\right] \mathrm{d} t\right\} .
\end{aligned}
$$

Remark 7. The function $Q(a, b ; p, q ; z)$ defined by (5) can be rewritten as

$$
Q(a, b ; p, q ; z)=\frac{\Gamma(b)}{\Gamma(a)}\left(\frac{b}{a}\right)^{(q-p+1) z} \frac{G(b, q, z)}{G(a, p, z)},
$$

where

$$
G(c, r, z)=\frac{[\Gamma(z+1)]^{r}}{\Gamma(r z+c)} .
$$

Taking the logarithm of $G(c, r, z)$ and differentiating gives

$$
\ln G(c, r, z)=r \ln \Gamma(z+1)-\ln \Gamma(r z+c)
$$

and

$$
\frac{\mathrm{d}}{\mathrm{d} z}[\ln G(c, r, z)]=r[\psi(z+1)-\psi(r z+c)] .
$$

Therefore, we obtain

$$
\begin{aligned}
\frac{\mathrm{d}}{\mathrm{d} z}\left[\ln \frac{G(b, q, z)}{G(a, p, z)}\right] & =q[\psi(z+1)-\psi(q z+b)]-p[\psi(z+1)-\psi(p z+a)] \\
& =(q-p) \psi(z+1)-[q \psi(q z+b)-p \psi(p z+a)]
\end{aligned}
$$


and

$$
\frac{\mathrm{d}^{k+1}}{\mathrm{~d} z^{k+1}}\left[\ln \frac{G(b, q, z)}{G(a, p, z)}\right]=(q-p) \psi^{(k)}(z+1)-q^{k+1} \psi^{(k)}(q z+b)+p^{k+1} \psi^{(k)}(p z+a)
$$

for $k \in\{0\} \cup \mathbb{N}$. Further making use of (15) arrives at

$$
(-1)^{k+1} \frac{\mathrm{d}^{k+1}}{\mathrm{~d} z^{k+1}}\left[\ln \frac{G(b, q, z)}{G(a, p, z)}\right]=\int_{0}^{\infty} \frac{t^{k}}{1-e^{-t}}\left[(q-p) e^{-(z+1) t}-q^{k+1} e^{-(q z+b) t}+p^{k+1} e^{-(p z+a) t}\right] \mathrm{d} t
$$

for $k \in \mathbb{N}$. Consequently, for $k \in \mathbb{N}$, we have

$$
(-1)^{k+1} \frac{\mathrm{d}^{k+1} \ln Q(a, b ; p, q ; z)}{\mathrm{d} z^{k+1}}=\int_{0}^{\infty} \frac{t^{k}}{1-e^{-t}}\left[(q-p) e^{-(z+1) t}-q^{k+1} e^{-(q z+b) t}+p^{k+1} e^{-(p z+a) t}\right] \mathrm{d} t
$$

\section{Properties of the Fuss-Catalan-Qi Function}

Recall from ([35] Chapter XIII), ([36] Chapter 1), and ([37] Chapter IV) that an infinitely differentiable function $f$ is said to be completely monotonic on an interval $I$ if it satisfies $0 \leq$ $(-1)^{k} f^{(k)}(x)<\infty$ on $I$ for all $k \geq 0$.

Recall from [38,39] that an infinitely differentiable and positive function $f$ is said to be logarithmically completely monotonic on an interval $I$ if $0 \leq(-1)^{k}[\ln f(x)]^{(k)}<\infty$ holds on $I$ for all $k \in \mathbb{N}$. For more information on logarithmically completely monotonic functions, please refer to [40-43] and plenty of references therein.

Recall from [38] that if $f^{(k)}(x)$ for some nonnegative integer $k$ is completely monotonic on an interval $I$ but $f^{(k-1)}(x)$ is not completely monotonic on $I$, then $f(x)$ is called a completely monotonic function of the $k$-th order on an interval $I$.

Stimulated by the above definitions and main results in [44], we now introduce the concept of logarithmically completely monotonic functions of the $k$-th order.

Definition 1. For a positive function $f(x)$ on an interval $I$, if $[\ln f(x)]^{(k)}$ for some nonnegative integer $k$ is completely monotonic on an interval I but $[\ln f(x)]^{(k-1)}$ is not completely monotonic on $I$, then we call $f(x)$ a logarithmically completely monotonic function of the $k$-th order on $I$.

In terms of the terminology of logarithmically completely monotonic functions of the $k$-th order, we can state the main results of this section as the following theorem.

Theorem 5. The function

$$
Q(a, b ; q, q ; x)=\frac{\Gamma(b)}{\Gamma(a)}\left(\frac{b}{a}\right)^{x} \frac{\Gamma(q x+a)}{\Gamma(q x+b)}, \quad a, b, p>0, \quad x \geq 0
$$

satisfies the following conclusions:

1. if $a<b$ and $q \leq \frac{\ln b-\ln a}{\psi(b)-\psi(a)}$, the function $Q(a, b ; q, q ; x)$ is increasing on $[0, \infty)$;

2. if $a>b$ and $q \leq \frac{\ln b-\ln a}{\psi(b)-\psi(a)}$, the function $Q(a, b ; q, q ; x)$ is decreasing on $[0, \infty)$;

3. If $a<b$ and $q>\frac{\ln b-\ln a}{\psi(b)-\psi(a)}$, the function $Q(a, b ; q, q ; x)$ has a unique minimum on $(0, \infty)$;

4. if $a>b$ and $q>\frac{\ln b-\ln a}{\psi(b)-\psi(a)}$, the function $Q(a, b ; q, q ; x)$ has a unique maximum on $(0, \infty)$;

5. if and only if $a \lessgtr b$, the function $[Q(a, b ; q, q ; x)]^{ \pm}$is logarithmically completely monotonic of the second order on $[0, \infty)$; in particular, if and only if $a \lessgtr b$, the function $[Q(a, b ; q, q ; x)]^{ \pm 1}$ is logarithmically convex on $[0, \infty)$.

Proof. Taking the logarithm on both sides of Equation (5) and differentiating with respect to $x$ yields

$$
\frac{\mathrm{d}[\ln Q(a, b ; p, q ; x)]}{\mathrm{d} x}=(q-p+1) \ln \frac{b}{a}+(q-p) \psi(x+1)+p \psi(p x+a)-q \psi(q x+b)
$$


and

$$
\frac{\mathrm{d}^{2}[\ln Q(a, b ; p, q ; x)]}{\mathrm{d} x^{2}}=(q-p) \psi^{\prime}(x+1)+p^{2} \psi^{\prime}(p x+a)-q^{2} \psi^{\prime}(q x+b) .
$$

It is not difficult to see that

$$
\lim _{x \rightarrow 0^{+}} \frac{\mathrm{d}[\ln Q(a, b ; p, q ; x)]}{\mathrm{d} x}=(q-p+1) \ln \frac{b}{a}+(q-p) \psi(1)+p \psi(a)-q \psi(b)
$$

and

$$
\begin{aligned}
& \lim _{x \rightarrow \infty} \frac{\mathrm{d}[\ln Q(a, b ; p, q ; x)]}{\mathrm{d} x}=(q-p+1) \ln \frac{b}{a}+\lim _{x \rightarrow \infty}\{(q-p)[\ln (x+1) \\
& \left.-\frac{1}{2(x+1)}-\sum_{n=1}^{\infty} \frac{B_{2 n}}{2 n(x+1)^{2 n}}\right]+p\left[\ln (p x+a)-\frac{1}{2(p x+a)}\right. \\
- & \left.\left.\sum_{n=1}^{\infty} \frac{B_{2 n}}{2 n(p x+a)^{2 n}}\right]-q\left[\ln (q x+b)-\frac{1}{2(q x+b)}-\sum_{n=1}^{\infty} \frac{B_{2 n}}{2 n(q x+b)^{2 n}}\right]\right\} \\
= & (q-p+1) \ln \frac{b}{a}+\lim _{x \rightarrow \infty}[(q-p) \ln (x+1)+p \ln (p x+a)-q \ln (q x+b)] \\
+ & \lim _{x \rightarrow \infty}\left\{(p-q)\left[\frac{1}{2(x+1)}+\sum_{n=1}^{\infty} \frac{B_{2 n}}{2 n(x+1)^{2 n}}\right]\right. \\
- & \left.p\left[\frac{1}{2(p x+a)}+\sum_{n=1}^{\infty} \frac{B_{2 n}}{2 n(p x+a)^{2 n}}\right]+q\left[\frac{1}{2(q x+b)}+\sum_{n=1}^{\infty} \frac{B_{2 n}}{2 n(q x+b)^{2 n}}\right]\right\} \\
= & (q-p+1) \ln \frac{b}{a}+\lim _{x \rightarrow \infty}[(q-p) \ln (x+1)+p \ln (p x+a)-q \ln (q x+b)] \\
= & (q-p+1) \ln \frac{b}{a}+p \ln p-q \ln q \\
= & \ln \left[\left(\frac{b}{a}\right)^{q-p+1} \frac{p^{p}}{q^{q}}\right],
\end{aligned}
$$

where the asymptotic expansion

$$
\psi(z) \sim \ln z-\frac{1}{2 z}-\sum_{n=1}^{\infty} \frac{B_{2 n}}{2 n z^{2 n}}
$$

as $z \rightarrow \infty$ in $|\arg z|<\pi$ (see [33] p. 259, 6.3.18) was used, and $B_{k}$ stands for the Bernoulli numbers that are defined by

$$
\frac{x}{e^{x}-1}=\sum_{k=0}^{\infty} \frac{B_{k} x^{k}}{k !}
$$

When $p=q$, making use of

$$
\psi^{(k)}(z)=(-1)^{k+1} \int_{0}^{\infty} \frac{t^{k}}{1-e^{-t}} e^{-z t} \mathrm{~d} t, \quad \Re(z)>0, \quad k \in \mathbb{N}
$$

in ([33] p. 260, 6.4.1) leads to

$$
\frac{\mathrm{d}^{2}[\ln Q(a, b ; q, q ; x)]}{\mathrm{d} x^{2}}=q^{2}\left[\psi^{\prime}(q x+a)-\psi^{\prime}(q x+b)\right]=q^{2} \int_{0}^{\infty} \frac{t}{1-e^{-t}} e^{-q x t}\left(e^{-a t}-e^{-b t}\right) \mathrm{d} t,
$$


which means that the derivative $\pm \frac{\mathrm{d}^{2}[\ln Q(a, b ; q, q ; x)]}{\mathrm{d} x^{2}}$ is completely monotonic on $[0, \infty)$ if and only if $a \lessgtr b$. Hence, the first derivative $\pm \frac{\mathrm{d}[\ln Q(a, b ; q, q ; x)]}{\mathrm{d} x}$ is increasing on $[0, \infty)$ if and only if $a \lessgtr b$. Meanwhile, the limits (13) and (14) become

$$
\lim _{x \rightarrow 0^{+}} \frac{\mathrm{d}[\ln Q(a, b ; q, q ; x)]}{\mathrm{d} x}=\ln \frac{b}{a}+q[\psi(a)-\psi(b)]=[\psi(a)-\psi(b)]\left[q-\frac{\ln b-\ln a}{\psi(b)-\psi(a)}\right]
$$

and

$$
\lim _{x \rightarrow \infty} \frac{\mathrm{d}[\ln Q(a, b ; q, q ; x)]}{\mathrm{d} x}=\ln \frac{b}{a}
$$

As a result,

1. if $a<b$ and $q \leq \frac{\ln b-\ln a}{\psi(b)-\psi(a)}$, the first derivative $\frac{\mathrm{d}[\ln Q(a, b ; q, q ; x)]}{\mathrm{d} x}$ is non-negative on $[0, \infty)$;

2. if $a<b$ and $q>\frac{\ln b-\ln a}{\psi(b)-\psi(a)}$, the first derivative $\frac{\mathrm{d}[\ln Q(a, b ; q, q ; x)]}{\mathrm{d} x}$ has a unique zero, which is a minimum point of $\ln Q(a, b ; q, q ; x)$, on $(0, \infty)$;

3. if $a>b$ and $q \leq \frac{\ln b-\ln a}{\psi(b)-\psi(a)}$, the first derivative $\frac{\mathrm{d}[\ln Q(a, b ; q, q ; x)]}{\mathrm{d} x}$ is non-positive on $[0, \infty)$;

4. if $a>b$ and $q>\frac{\ln b-\ln a}{\psi(b)-\psi(a)}$, the first derivative $\frac{\mathrm{d}[\ln Q(a, b ; q, q ; x)]}{\mathrm{d} x}$ has a unique zero, which is a maximum point of $\ln Q(a, b ; q, q ; x)$, on $(0, \infty)$.

Therefore, the conclusions on $Q(a, b ; q, q ; x)$ are thus proved. The proof of Theorem 5 is complete.

\section{Remarks}

Finally, we list additional several remarks.

Remark 8. Combining Theorem 5 and the last relation in (6), we obtain that the Catalan-Qi function $C(a, b ; x)$ is a logarithmically completely monotonic function of the second order.

Remark 9. Similar to the introduction of the Catalan-Qi function $C(a, b ; z)$ in [22], we had better leave the combinatorial interpretation of the Fuss-Catalan-Qi function $Q(a, b ; p, q ; z)$ to combinatorialists and number theorists.

Remark 10. In recent years, there were many results on the Catalan numbers $C_{n}$ and the Catalan-Qi numbers $C(a, b ; n)$ in $[45-53]$ and the closely related references therein.

Remark 11. This paper is a corrected and revised version of the preprint [54].

\section{Conclusions}

In this paper, we introduce a unified generalization of the Catalan numbers, the Fuss numbers, the Fuss-Catalan numbers, and the Catalan-Qi function, and discover some properties of the unified generalization, including a product-ratio expression of the unified generalization in terms of the Catalan-Qi functions, three integral representations of the unified generalization, and the logarithmically complete monotonicity of the second order for a special case of the unified generalization.

Author Contributions: The authors contributed equally to this work. All authors read and approved the final manuscript.

Funding: This research received no external funding.

Acknowledgments: The authors thank anonymous referees for their careful corrections to and valuable comments on the original version of this paper.

Conflicts of Interest: The authors declare no conflict of interest. 


\section{References}

1. Koshy, T. Catalan Numbers with Applications; Oxford University Press: Oxford, UK, 2009.

2. Stanley, R.P. Catalan Numbers; Cambridge University Press: New York, NY, USA, 2015.

3. Stanley, R.P. Enumerative Combinatorics; Cambridge University Press: Cambridge, UK, 1999.

4. Graham, R.L.; Knuth, D.E.; Patashnik, O. Concrete Mathematics-A Foundation for Computer Science, 2nd ed.; Addison-Wesley Publishing Company: Reading, MA, USA, 1994.

5. Vardi, I. Computational Recreations in Mathematica; Addison-Wesley: Redwood City, CA, USA, 1991.

6. Qi, F.; Mahmoud, M.; Shi, X.-T.; Liu, F.-F. Some properties of the Catalan-Qi function related to the Catalan numbers. SpringerPlus 2016, 5, 1126. [CrossRef]

7. Elezović, N. Asymptotic expansions of gamma and related functions, binomial coefficients, inequalities and means. J. Math. Inequal. 2015, 9, 1001-1054. [CrossRef]

8. Hilton, P.; Pedersen, J. Catalan numbers, their generalization, and their uses. Math. Intell. 1991, 13, 64-75. [CrossRef]

9. Klarner, D.A. Correspondences between plane trees and binary sequences. J. Combin. Theory 1970, 9, 401-411. [CrossRef]

10. Fuss, N.I. Solutio quaestionis, quot modis polygonum $\mathrm{n}$ laterum in polygona $\mathrm{m}$ laterum, per diagonales resolvi queat. Nova Acta Acad. Sci. Imp. Petropolitanae 1791, 9, 243-251.

11. Alexeev, N.; Götze, F.; Tikhomirov, A. Asymptotic distribution of singular values of powers of random matrices. Lith. Math. J. 2010, 50, 121-132. [CrossRef]

12. Aval, J.-C. Multivariate Fuss-Catalan numbers. Discret. Math. 2008, 308, 4660-4669. [CrossRef]

13. Gordon, I.G.; Griffeth, S. Catalan numbers for complex reflection groups. Am. J. Math. 2012, 134, 1491-1502. [CrossRef]

14. Bisch, D.; Jones, V. Algebras associated to intermediate subfactors. Invent. Math. 1997, 128, 89-157.

15. Lin, C.-H. Some combinatorial interpretations and applications of Fuss-Catalan numbers. ISRN Discret. Math. 2011, 2011, 534628. [CrossRef]

16. Liu, D.-Z.; Song, C.-W.; Wang, Z.-D. On explicit probability densities associated with Fuss-Catalan numbers. Proc. Am. Math. Soc. 2011, 139, 3735-3738. [CrossRef]

17. Młotkowski, W. Fuss-Catalan numbers in noncommutative probability. Doc. Math. 2010, 15, 939-955.

18. Młotkowski, W.; Penson, K.A.; Zyczkowski, K. Densities of the Raney distributions. Doc. Math. 2013, 18, 1573-1596.

19. Przytycki, J.H.; Sikora, A.S. Polygon dissections and Euler, Fuss, Kirkman, and Cayley numbers. J. Combin. Theory Ser. A 2000, 92, 68-76. [CrossRef]

20. Stump, C. $q, t$-Fuß-Catalan numbers for complex reflection groups. In Proceedings of the 20th Annual International Conference on Formal Power Series and Algebraic Combinatorics (FPSAC 2008), Valparaiso, Chile, 23-27 June 2008; pp. 295-306.

21. Stump, C. q, t-Fuß-Catalan numbers for finite reflection groups. J. Algebr. Combin. 2010, 32, 67-97. [CrossRef]

22. Qi, F.; Shi, X.-T.; Mahmoud, M.; Liu, F.-F. The Catalan numbers: A generalization, an exponential representation, and some properties. J. Comput. Anal. Appl. 2017, 23, 937-944.

23. Qi, F.; Cerone, P. Some properties of the Fuss-Catalan numbers. Mathematics 2018, 6, 277. [CrossRef]

24. Qi, F.; Shi, X.-T.; Liu, F.-F. An integral representation, complete monotonicity, and inequalities of the Catalan numbers. Filomat 2018, 32, 575-587. [CrossRef]

25. Liu, F.-F.; Shi, X.-T.; Qi, F. A logarithmically completely monotonic function involving the gamma function and originating from the Catalan numbers and function. Glob. J. Math. Anal. 2015, 3, 140-144. [CrossRef]

26. Mahmoud, M.; Qi, F. Three identities of the Catalan-Qi numbers. Mathematics 2016, 4, 35. [CrossRef]

27. Qi, F.; Guo, B.-N. Logarithmically complete monotonicity of a function related to the Catalan-Qi function. Acta Univ. Sapientiae Math. 2016, 8, 93-102. [CrossRef]

28. Qi, F.; Guo, B.-N. Logarithmically complete monotonicity of Catalan-Qi function related to Catalan numbers. Cogent Math. 2016, 3, 1179379. [CrossRef]

29. Qi, F.; Guo, B.-N. Some properties and generalizations of the Catalan, Fuss, and Fuss-Catalan numbers. Mathematical Analysis and Applications: Selected Topics, 1st ed.; Ruzhansky, M., Dutta, H., Agarwal, R.P., Eds.; John Wiley \& Sons, Inc.: Hoboken, NJ, USA, 2018; Chapter 5, pp. 101-133. 
30. Qi, F.; Shi, X.-T.; Liu, F.-F.; Kruchinin, D.V. Several formulas for special values of the Bell polynomials of the second kind and applications. J. Appl. Anal. Comput. 2017, 7, 857-871.

31. Qi, F.; Shi, X.-T.; Mahmoud, M.; Liu, F.-F. Schur-convexity of the Catalan-Qi function related to the Catalan numbers. Tbilisi Math. J. 2016, 9, 141-150. [CrossRef]

32. Shi, X.-T.; Liu, F.-F.; Qi, F. An integral representation of the Catalan numbers. Glob. J. Math. Anal. 2015, 3, 130-133. [CrossRef]

33. Abramowitz, M.; Stegun, I.A. Handbook of Mathematical Functions with Formulas, Graphs, and Mathematical Tables; National Bureau of Standards: Washington, DC, USA, 1972.

34. Temme, N.M. Special Functions: An Introduction to Classical Functions of Mathematical Physics; John Wiley \& Sons, Inc.: New York, NY, USA, 1996.

35. Mitrinović, D.S.; Pečarić, J.E.; Fink, A.M. Classical and New Inequalities in Analysis; Kluwer Academic Publishers: Dordrecht, The Netherlands, 1993.

36. Schilling, R.L.; Song, R.; Vondraček, Z. Bernstein Functions-Theory and Applications, 2nd ed.; Walter de Gruyter: Berlin, Germany, 2012.

37. Widder, D.V. The Laplace Transform; Princeton University Press: Princeton, NJ, USA, 1941.

38. Atanassov, R.D.; Tsoukrovski, U.V. Some properties of a class of logarithmically completely monotonic functions. Dokladi na Bolgarskata Akademiya na Naukite 1988, 41, 21-23.

39. Qi, F.; Chen, C.-P. A complete monotonicity property of the gamma function. J. Math. Anal. Appl. 2004, 296, 603-607. [CrossRef]

40. Qi, F.; Guo, S.; Guo, B.-N. Complete monotonicity of some functions involving polygamma functions. J. Comput. Appl. Math. 2010, 233, 2149-2160. [CrossRef]

41. Qi, F.; Li, W.-H. A logarithmically completely monotonic function involving the ratio of gamma functions. J. Appl. Anal. Comput. 2015, 5, 626-634.

42. Qi, F.; Luo, Q.-M.; Guo, B.-N. Complete monotonicity of a function involving the divided difference of digamma functions. Sci. China Math. 2013, 56, 2315-2325. [CrossRef]

43. Qi, F.; Wei, C.-F.; Guo, B.-N. Complete monotonicity of a function involving the ratio of gamma functions and applications. Banach J. Math. Anal. 2012, 6, 35-44. [CrossRef]

44. Guo, B.-N.; Qi, F. A property of logarithmically absolutely monotonic functions and the logarithmically complete monotonicity of a power-exponential function. Politehn. Univ. Bucharest Sci. Bull. Ser. A Appl. Math. Phys. 2010, 72, 21-30.

45. Yin, L.; Qi, F. Several series identities involving the Catalan numbers. Trans. A. Razmadze Math. Inst. 2018, 172, 466-474. [CrossRef]

46. Qi, F. An improper integral, the beta function, the Wallis ratio, and the Catalan numbers. Probl. Anal. Issues Anal. 2018, 7, 104-115. [CrossRef]

47. Qi, F. Parametric integrals, the Catalan numbers, and the beta function. Elem. Math. 2017, 72, $103-110$. [CrossRef]

48. Qi, F. Some properties of the Catalan numbers. Ars Combin. 2022, in press. Available online: https: / / www.researchgate.net/publication/328891537 (accessed on 4 May 2019).

49. Qi, F.; Akkurt, A.; Yildirim, H. Catalan numbers, k-gamma and $k$-beta functions, and parametric integrals. J. Comput. Anal. Appl. 2018, 25, 1036-1042.

50. Qi, F.; Guo, B.-N. Integral representations of the Catalan numbers and their applications. Mathematics 2017, 5, 40. [CrossRef]

51. Qi, F.; Zou, Q.; Guo, B.-N. Some identities and a matrix inverse related to the Chebyshev polynomials of the second kind and the Catalan numbers. Available online: https:/ / doi.org/10.20944/preprints201703.0209.v2 (accessed on 4 May 2019).

52. Zou, Q. Analogues of several identities and supercongruences for the Catalan-Qi numbers. J. Inequal. Spec. Funct. 2016, 7, 235-241. 
53. Zou, Q. The $q$-binomial inverse formula and a recurrence relation for the $q$-Catalan-Qi numbers. J. Math. Anal. 2017, 8, 176-182.

54. Qi, F.; Shi, X.-T.; Cerone, P. A unified generalization of the Catalan, Fuss, Fuss-Catalan numbers and Catalan-Qi function. Available online: https:/ / doi.org/10.13140/RG.2.1.3198.6000 (accessed on 4 May 2019).

(C) 2019 by the authors. Licensee MDPI, Basel, Switzerland. This article is an open access article distributed under the terms and conditions of the Creative Commons Attribution (CC BY) license (http:/ / creativecommons.org/licenses/by/4.0/). 